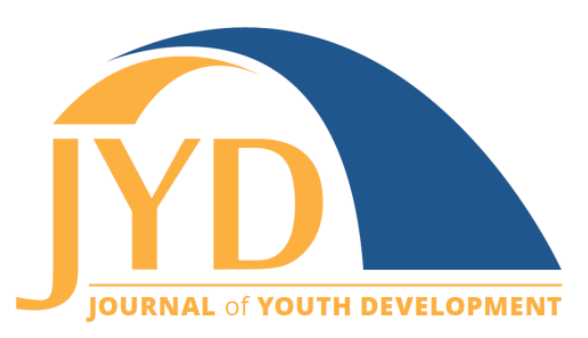

http://jyd. pitt. edu/ | Vol. 14 Issue 1 DOI 10.5195/jyd.2019.699 | ISSN 2325-4017 (online)

\title{
Strategies for Improving Self-Efficacy: A Qualitative Analysis of Detroit's Downtown Boxing Gym
}

\author{
Amanda Sommerfeld Case \\ Purdue University \\ amandacase@purdue.edu \\ N. Keita Christophe \\ University of North Carolina, Greensboro \\ nnchrist@uncg.edu
}

\begin{abstract}
Structural inequalities present throughout U.S. public schools are known to contribute to the significant achievement gaps that persist between lower-income students of color and their more financially secure, White peers. Because of this, community programs have been identified as places where typically underserved students can receive the support required for positive development and academic achievement. The current study used qualitative methods to explore how one community program, Detroit's Downtown Boxing Gym, fosters self-efficacy in school-aged youth from Detroit Public Schools. Focus group participants reported they are indeed experiencing increases in self-efficacy as a result of the mastery experiences, vicarious experiences, and verbal persuasion they receive at the gym. Specific recommendations for how other programs might foster self-efficacy, including establishing a program climate where students feel cared for, ensuring program staff truly believe students can be successful, identifying opportunities for students to have mastery experiences, and utilizing peer modeling, are discussed.
\end{abstract}

Key words: community youth development, self-efficacy, lower-income youth of color, qualitative research

Structural inequalities within U.S. public schools, such as lack of funding, overcrowded classrooms, outdated textbooks, and little access to extracurricular activities (DarlingHammond, 2001), are making it more difficult for many lower-income students of color to succeed academically (Nickens \& Smedley, 2001). Because of these inequities, programming

(cc) $\mathbf{E Y}$ New articles in this journal are licensed under a Creative Commons Attribution 4.0 License. This journal is published by the University Library System, University of Pittsburgh and is cosponsored by the University of Pittsburgh Press. The Journal of Youth Development is the official peer-reviewed publication of the National Association of Extension 4-H Agents and the National AfterSchool Association. 
Improving Self-Efficacy Via Community Programs

outside of schools needs to be available that targets factors that can reduce risk, promote resilience, and facilitate educational achievement in youth (Berg, Coman, \& Schensul, 2009; Schwartz \& Suyemoto, 2013). One such factor is self-efficacy, which is correlated with numerous positive academic outcomes in students (Britner \& Pajares, 2006; Robbins et al., 2004). To date, research has not consistently demonstrated what aspects of youth development programming most effectively increase self-efficacy beliefs in students. As a result, youth development programs have often been left with limited guidance about how to foster selfefficacy in their students. The purpose of this qualitative study is to learn from the youth involved in one community-based program in order to improve understandings of how youth development programs across the country can support the efficacy development of lowerincome youth of color.

\section{What is Self-Efficacy?}

Self-efficacy is defined as a person's judgment that he or she can successfully execute the behavior required to produce a desired outcome, even in the face of adversity (Bandura, 1977). These judgments can be both general and domain-specific (Schunk \& Pajares, 2002). For example, one may have high general self-efficacy beliefs, resulting in an overarching belief that he or she can overcome challenges and experience success on most tasks. However, one's domain-specific self-efficacy may be lower for certain tasks, such as math, causing doubt in his or her ability to be successful on tasks requiring math skills. Researchers have consistently documented the wide-ranging and influential effects general and domain-specific self-efficacy beliefs have, finding high self-efficacy beliefs to be associated with higher educational and career aspirations, better performance regardless of ability level, greater resilience, and decreased vulnerability to stress and depression (Alvernini \& Lucidi, 2011; Bandura, Barbaranelli, Caprara, \& Pastorelli, 2001). This is especially the case for systematically disadvantaged youth, for whom self-efficacy may serve a protective role (Bandura \& Cervone, 1983; DeAngelis, 2012). Low self-efficacy beliefs, on the other hand, are associated with opposite, negative outcomes such as lower aspirations and decreased motivation, performance deterioration, and increased manifestation of depression and anxiety symptoms (Bandura et al., 2001; Butz \& Usher, 2015; Samuels \& Gibb, 2002). 


\section{Effectiveness of Community-Based Programs in Building Self-Efficacy}

Bandura (1982) theorized four ways people can increase self-efficacy: mastery experience, verbal persuasion, vicarious experience, and physiological state. Butz and Usher (2015) briefly describe these four methods, saying "Beliefs about one's . . . capabilities are formed and modified as individuals interpret their past experiences (mastery experience), the messages that they receive from others (verbal persuasion), what they see others do (vicarious experience), and how they feel while engaged in or thinking about an activity (physiological and affective states)" (p. 49).

Although Bandura (1982) clearly articulated mechanisms for increasing self-efficacy, community youth development programs have not been consistently successful in putting theory into practice and demonstrating how their programming affects student self-efficacy (Morton \& Montgomery, 2013). For example, Morton and Montgomery (2013) conducted a meta-analysis to examine the efficacy-building effects of three community youth programs. The programs included in the study were designed to increase self-efficacy beliefs, amongst other psychosocial factors, by giving students participatory roles in program activities and empowering them to make meaningful program decisions. The results of their analyses suggested that the programs did not reliably induce increases in domain-specific or general selfefficacy. Berg and colleagues (2009) also conducted a similar analysis of community-based programs designed to increase self-efficacy in children and found, unfortunately, that these programs were also ineffective in increasing self-efficacy beliefs.

Despite results suggesting that programs are not successfully increasing participant selfefficacy, researchers are not yet convinced that these programs are wholly ineffective. Instead, researchers point to methodological limitations that may have obfuscated program effectiveness. For example, both Berg and colleagues (2009) and Morton and Montgomery (2013) claimed that the lack of evidence about the benefits of community programs in increasing self-efficacy may be due to poor experimental designs and shortcomings in the instruments used to measure self-efficacy. As a result, Berg and colleagues called for not only an increase in the number and quality of quantitative investigations about the effectiveness of efficacy-building community programs but also for the use of qualitative approaches.

As Usher and Pajares (2008) posit, qualitative approaches may be better suited to studying the effects of efficacy-building programs because they allow researchers to more deeply understand the genesis of efficacy-related beliefs while also considering students' specific contexts, stages 
of development, and other personal considerations. Therefore, by conducting qualitative analyses of programs aiming to increase self-efficacy, researchers may be better able to accurately reveal the positive effects program participation may have on youths' self-efficacy beliefs (Cervone, 1997).

\section{Current Study}

Because of the important role that self-efficacy plays in academic outcomes-especially for systematically disadvantaged students-identifying features of community-based programs that support self-efficacy development would go a long way in promoting educational success in lower-income students of color. Up to this point, however, analyses of self-efficacy development in youth programming have been hampered by methodological constraints. As a result, gaps still exist in what is known about how community-based programs might enhance self-efficacy development. The current study aims to address these gaps by examining how one communitybased youth development program, the Downtown Boxing Gym (DBG), fosters self-efficacy development in the lower-income youth of color that it serves.

The DBG is a community-based program that provides boxing instruction, mentoring, and comprehensive tutoring, all free of charge to students from Detroit. Although the program is open to all students regardless of race or socioeconomic background, the program has historically served all students of color, approximately $99 \%$ of whom come from lower-income households. Since it opened in 2007, DBG has served over 500 youth, approximately $35 \%$ of whom are still enrolled in the program and more than $60 \%$ of whom have graduated from the program. Of those graduates, $100 \%$ have graduated from high school and been accepted to a 2-year or 4-year college or university. Because the gym is clearly having a positive effect on students enrolled in the program, the gym provides a good opportunity to explore whether and how this community intervention is supporting the development of self-efficacy beliefs in youth.

\section{Methods}

\section{Data Collection}

Six semi-structured focus groups were conducted to explore how participation in DBG affected the self-efficacy of study participants. As part of a longitudinal investigation of the gym that is examining the consistency of program effects over time, data were collected at two time points with separate participants; three focus groups were conducted in spring 2013 and another three 
were conducted in summer 2016. The first author conducted all three of the focus groups in spring 2013. Both the first and second authors, along with another member of the research team, conducted focus groups in summer 2016. All focus groups were structured around four main topics: the services the gym offers, how participants believe they have been affected by participating in the gym, what specific aspects of the gym caused the participants to be affected in the way they were, and how the gym can improve. In keeping with the aims of semistructured interviews, follow-up questions were also asked on these topics and participants were encouraged to bring up novel topics that were related to their experiences at the gym.

All focus groups were held at DBG, in a room where only research team members and participants were present. All focus groups were audio- and video-recorded. Before initiating the discussion, the researchers introduced themselves and reviewed the purpose of the focus group, which was described as "learning what the gym is all about, what you do here, and what you've learned since you started coming here." Participants were then informed of the "rules" of the focus group, including that "no topic is off limits," students do not have to raise their hands and can just "jump in" when they have something to share, and that responses would remain confidential. Participants under the age of 18 then signed an assent form (consent forms were signed by participants aged 18 years or older), after which the participants were asked to share their name, age, and how long they had been coming to the gym. Focus groups lasted for between 30 and 50 minutes, with the duration being determined by the participants.

\section{Participants}

Focus groups ranged in size from four to 11 participants. The executive director and academic coordinator of DBG were responsible for deciding which students would participate in which focus groups. The composition of the focus groups was mainly determined by participant availability; however, one focus group was intentionally comprised of only girls and another was made up of younger students (i.e., less than 14 years of age). This decision was made so students who are underrepresented in the gym had a comfortable space to share their experiences.

A total of 49 students participated in the focus groups, which lasted an average of 35 minutes each. Participants included 28 males and 21 females. Most of the participants $(N=45)$ identified as African American, with the remaining four identifying as Latino. Ages of participants ranged from 8 to 20 years old, with the majority of participants being between the ages of 12 and 19. Although DBG chooses not to collect specific income data from individual 
students and families, based on known individual, neighborhood, and school data, program staff estimated that all of the participants qualified for free lunch at school. The average length of time participants had been involved in the gym at the time of participation in the focus group was $1 \frac{1}{2}$ years, though the length of involvement varied greatly from a few weeks to several years.

\section{Data Analysis}

The second author and members of the research team transcribed the audio recordings of the focus groups verbatim. The first and second authors then analyzed the transcriptions using a thematic analysis approach. Thematic analysis is a flexible approach that "involves the search for and identification of common threads that extend across an entire interview or set of interviews" (Vaismoradi, Turunen, \& Bondas, 2013, p. 400). In keeping with the thematic analysis phases outlined by Braun and Clarke (2006), the authors conducted the analysis of focus group data in five steps. First, we familiarized ourselves with the data by reading and rereading the transcriptions in their entirety. After we felt sufficiently comfortable with the data, we generated initial codes for notable features of the data and collated the data relevant to each code. Third, to provide a detailed account of data relevant to self-efficacy, we pulled together all of the codes and exemplar data relevant to this theme. Once gathered, these codes and exemplars were checked to ensure they made sense in the context of the entire data set. All of the data thematically related to self-efficacy was then further analyzed to refine the subcategories of the theme and identify areas of overlap with Bandura's theory. Finally, we selected examples from the data that vividly captured the nuances related to the theme of selfefficacy development.

\section{Results}

During the focus groups most of the participants demonstrated considerable self-reflectiveness in describing their experiences in the gym and the ways they had changed as a result of those experiences. Mimicking the gym's mission, which emphasizes academics, personal and social responsibility, and preparing for the future, many of the participants highlighted their personal growth in these areas. For example, many spoke about the academic improvements they had made in school and several noted beneficial social and behavioral growth as well. Participants also spoke at length about transformations they experienced in how they thought about themselves and their abilities. More specifically, although the students never explicitly used the term self-efficacy, their narratives were nonetheless interspersed with numerous examples of 
how participating in the gym resulted in increases in their self-efficacy beliefs. The examples they shared suggested there are three primary mechanisms through which the gym increased their self-efficacy: mastery experiences, vicarious experiences, and verbal persuasion.

\section{Mastery Experiences}

Mastery experiences are instances in which a person successfully completes a task or overcomes a challenge, leading him or her to feel more capable of doing so again in the future (Bandura, 1982). Participants reported experiencing many such instances at the gym with regard to boxing and school. Often these descriptions were framed in terms of self-discipline, with numerous participants describing that because they had learned to "control" themselves inside the gym, they were also able to do so outside of the gym. As one participant said, "I feel like you can't turn it off. So it's not like I could come here, learn how to be a nice person [and then] go out into the world and not do that." Another student agreed, saying "how you act here is, like, how you act outside of the gym."

Many students attributed the mastery they had over themselves to the boxing training they received. For example, one participant noted that "in boxing they teach you to control your anger." As a result, as another student described, that sense of self-control flows into other settings as well: "I think [learning to box] helps out a lot, especially in school. Before I came here I [would] talk back to teachers. Now I'm a teacher's pet and I think [that's because of] the discipline [of boxing]. I live the discipline because now it's a habit for me." For many of the students, then, it seemed that the mastery experiences they had in the gym of being able to control themselves inside the ring allowed them to feel more able to control themselves outside of the ring in multiple settings as well.

Participants also described how the success they had at the gym when working on schoolwork allowed them to feel more capable of success overall, but especially in school. The gym is structured so each day students spend at least thirty minutes with an academic tutor before they can box. The gym's motto, "Books before Boxing," reinforces this prioritization. For the majority of the participants, it was in terms of their academics where they saw the greatest change in themselves. Almost unanimously the participants reported increases in their grades since joining the gym. But even beyond grades, many students also reported changes in their attitudes about school. As one participant bluntly stated, "I didn't care about school. I used to always sleep in class." But since working with the tutors at the gym, he realized that "for real, I like math!" In large part this change in attitude, which multiple participants echoed, seemed to 
grow out of a sense of mastery. For example, as another participant said, "the tutors, like, prepare you for what you gonna do in school." As a result of this preparation, many participants reported feeling more "confident" that they could be academically successful, which also caused them to feel as though their school work had gotten "much easier."

\section{Verbal Persuasion}

Verbal persuasion involves an increase in self-efficacy as a result of a person being told by others that he or she is able to overcome specific challenges. Many of the efficacy-building experiences participants talked about in the focus groups seemed to be due to some form of verbal persuasion coming from the adults in the gym. This verbal persuasion often arose naturally from the mentoring relationships adults in the gym worked hard to establish with the students. As one participant jokingly said, "the coaches, man, they always talking!" As a result of this "talking," participants reported feeling more capable of overcoming problems and completing tasks.

Many students named Coach Khali, the charismatic founder of DBG, as a primary source of verbal persuasion. For example, one participant told a story about an interaction he had with Khali after getting into an argument at school:

He was at the ring and I was sitting down and he's like, "See, you wanna achieve goals?" He's like, "My goal is to touch that [wall]that's my path and I'm gonna walk straight and keep walking straight. Say my name when I keep walking. "And I said "Khali!" and I kept saying it louder and louder and he was ignoring me and he touched the wall. And he was like, "See, I touched my goal without listening to anyone or letting them interfere in my path." He said "Now when I keep walking say something." After he kept walking I said something and he turned around and started walking back and said, "Yeah, what's up?" And he said "See what happens? I get off track and I start getting distracted by other people in my way cuz I listened to other people." He said "Just ignore people and do your own thing and go with your own path and achieve your goals."

Noteworthy in this example is the fact that Khali conveyed to the student that he was capable of achieving his goals. Many other students shared stories that suggested that everyone at the gym, including Khali, the other adults, and the students, all presumed each and every student 
could be successful physically, academically, and socially. As a result, the participants started to internalize this belief as well.

The participants also shared that the messages they received from people at the gym were especially powerful because of the gym's sense of community and also because most of the people at the gym shared certain life experiences. More specifically, every participant described the gym as a "family," and most mentioned that they felt "loved" by both the adults and their peers at the gym. Being told they were able to be successful from the members of that family, who they knew "cared" about them and also knew them "as individuals," left a lasting impression. In addition, several participants also described giving more weight to the encouragement they received from the adults and students in the gym because so many of them had "been through the same stuff." For example, several of the participants mentioned that the fact that several of the adults and students had been involved in negative activities in the "streets" made their messages that the students could do something more worthwhile with their lives all the more compelling.

\section{Vicarious Experience}

In addition to describing instances when their self-efficacy was being affected by mastery experiences and verbal persuasion, the students also shared numerous examples of how their self-efficacy beliefs were shaped through vicarious experiences. Vicarious experience involves seeing someone else succeed in a task, which can cause the observer to feel able to accomplish the task as well.

Students of all ages reported the power of observing their peers successfully navigate challenges. One student explained this, saying, "you come here and it's, like, different. It's people doing what they supposed to do. ... That's what makes you do what you supposed to do." Another student echoed this sentiment stating, "When you come here you just look at people and they kinda just motivate you and then you like 'Oh I need to get my act together."' Seeing peers thriving thus caused the students to feel as if they can also be successful if they mimicked their peers' behaviors.

Several students also talked about how their observations of peers led to transformative experiences. For example, one girl stated,

Before I started coming to this gym, I had a bad attitude. [But

here at the gym] there's different people here that set different 
Improving Self-Efficacy Via Community Programs

examples . . . Most of the people here, they graduated and made

it to college, so . . . that made me wanna go to college and

change my attitude and make me into a better person.

This student directly verbalized the effects of an efficacy-building vicarious experience. She saw others thriving at the gym and, as a result, started to believe that she, too, could succeed. Stories such as these suggest students in the gym are seeing their peers overcome challenges both inside and outside the walls of the gym, and these vicarious experiences are making them feel capable of overcoming those challenges as well.

Because the participants seemed to recognize the amount of influence the students have on one another, many of the older (i.e., high school) participants described feeling a sense of responsibility to set a "good example" for their younger peers. For some of these participants, doing so was "common sense." For others, however, they were told explicitly by their coaches that they needed to be positive role models for the younger students. Regardless of whether this understanding came naturally or via the adults at the gym, however, virtually all of the older participants conveyed that they understood the power of vicarious experience. As one older participant shared, "[the younger kids] see what we do and they'll do it. Like, if we do good in school then they'll wanna do good in school." For another participant, however, the benefits of setting positive examples are longstanding. As she said, "When we motivate [the younger kids], they will wanna motivate their kids, because they will think back to the day, like, oh yeah, I remember I looked up to that kid . . . and then they'll pass it on to generations." To the students in the gym, then, it seemed that seeing others try hard, overcome challenges, and be successful not only caused them to believe they could do the same, but also led them to try and set positive examples for others.

\section{Discussion}

Taken together, participant narratives suggested the structure of and activities in the gym helped them to feel more capable of success in many facets of their lives. In particular, the participants described an increased "confidence" in their abilities to manage their emotions and efforts, leading them to believe they could be successful both in school and in life. This strong relationship between self-efficacy and academic and life outcomes is well documented in the literature (Bandura, 1977, 1997). Less researched, however, is how programs can foster selfefficacy development in youth who are often the victims of structural inequalities. 
Students from DBG identified many salient mechanisms by which participating in the gym increased their self-efficacy. Experiences with the tutors at the gym were often cited as memorable and impactful, allowing students to experience scholastic success, cultivate a genuine interest in the subject matter, and perceive themselves as more capable in academic contexts. Adults in the gym also appeared to be a major mechanism by which student selfefficacy increased. Students perceived the adults in the gym as being very invested in them; instances of verbal persuasion where adults told students they were capable of success, therefore, seemed to help students feel able to succeed both inside and outside of the gym. Students in the gym who were successful academically, socially, and in the boxing ring also served as examples to their peers. Through watching these successful peers, participants reported vicarious experiences where they started feeling capable of similar success as well.

\section{Implications for Youth Development Programs}

Youth development programming is as diverse as the students it is intended to serve, which is highly beneficial; our students are not all the same, so we should not expect they would all benefit from the same interventions. However, based on the experiences of students who participated in the current study, it may be the case that there are certain qualities or practices that could be integrated into a variety of programs in order to foster self-efficacy development amongst systematically disadvantaged students. These qualities and practices include establishing a program climate where students feel cared for, ensuring program staff truly believe students can be successful, identifying opportunities for students to have mastery experiences, and utilizing peer modeling.

\section{Establishing a Caring Program Climate}

According to study participants, a large reason why they believed they were capable of success was because they were told they could be successful by people who knew them well and cared about them. In other words, the participants were receiving messages from people they considered credible (Bandura, 1997). As a result, when the adults at the gym told participants that they could be successful (i.e., when the participants received verbal persuasions about their self-efficacy), the participants heard and internalized those messages.

What these results point to is the supremacy of a program's culture in determining the effectiveness of efficacy-building efforts. If a program's culture allows students to feel safe, known, and respected, students may be more likely to believe positive messages about their 
potential. On the other hand, if the culture of the program does not foster such feelings of security or belonging, the potency of those messages may be diluted. Therefore, it may be worthwhile for programs to assess student perceptions of the program culture and, depending on the results of that assessment, either strategize about how a positive culture can be maintained or allocate resources to improve suboptimal cultures. For example, programs should set aside time to ensure all staff receiving ongoing training that reinforces the roles they play in creating a safe and respectful space in which they can develop meaningful relationships with students. Programs may also consider establishing a student advisory board, which both enables program staff to receive consistent feedback from students and signals to students that their voice is important to program functioning.

\section{Ensuring Staff Believe Students are Capable}

Adults endeavoring to support self-efficacy development in youth should believe those youth are capable of success. Such a statement may appear obvious; however, programs should not presume all staff think in this way. Research has consistently demonstrated that even adults who devote their lives to supporting youth hold implicit biases that cause them to underestimate student abilities, especially when the students are racial minorities or from lowerincome backgrounds (e.g., Dee \& Gershenson, 2017). These attitudes can have profoundly negative implications for student outcomes.

Thankfully, the reverse also seems to be true, in that being around adults who do believe students are capable can foster positive outcomes (e.g., Jussim \& Harber, 2005). This seemed to be the case for the participants in the current study. Although none of the participants explicitly stated that their self-efficacy increased because the adults at the gym believed they could succeed, many of the participants shared stories that suggested this was their experience. In fact, in various ways most of the participants intimated that the adults in the gym treated them as though success was inevitable as long as they remained dedicated to their goals.

Given these findings, programs may want to consider how they can ensure staff members and volunteers believe that the students they are working with can be successful, especially when those students are typically undervalued and underprivileged in American society. For example, though it may be logistically challenging, programs may want to require implicit bias training for all personnel. Recent research has also suggested that exposure to positive counter-stereotypes can reduce implicit bias (Devine, Forscher, Austin, \& Cox, 2012). As such, for the sake of both 
students and staff, program leaders may want to integrate discussions about stereotypes and counter-stereotypes into meetings.

\section{Identifying Opportunities for Students to Have Mastery Experiences.}

Bandura (1977) conceptualized self-efficacy beliefs as domain-specific and theorized that mastery experiences can only increase self-efficacy in the domain in which they occur. However, other scholars have argued for the existence of general self-efficacy beliefs that cut across domains and enable students to believe they are capable of overcoming challenges even in novel situations (Grether, Sowislo, \& Wiese, 2018; Schwartzer \& Jerusalem, 1995). These scholars suggest that mastery experiences in any domain could contribute to students' overall senses of whether or not they can be successful (e.g., Shelton, 1990). This seemed to be the experience of many of the study participants.

Multiple participants shared examples that suggested they had generalized the mastery experiences they had in the gym to life outside of the gym, especially at school. For these participants it seemed that what was most influential was not the particulars of the task on which they succeeded, but instead the fact that they had experienced success at all. Those experiences of success, then, seemed to transform how participants thought about their overall capabilities. These findings do not negate Bandura's notion that mastery experiences are domain-specific. However, they do suggest that experiencing any prosocial success may have far-reaching benefits. Therefore, program personnel may want to reflect on whether or not their program structure creates opportunities for students to be successful, even on small tasks. Ensuring this occurs may require programs to differentiate activities based on ages or abilities or to break larger tasks into component parts so students have many opportunities for mastery experiences.

\section{Utilizing Peer Modeling}

Virtually all of the participants in the current study described their peers as a key source of selfefficacy. In particular, many participants shared that seeing or hearing about their peers' successes inside or outside the gym caused them to feel that they, too, could be successful. According to Bandura (1977), these findings are not surprising. As he theorized, vicarious experiences are especially powerful sources of self-efficacy when the models that individuals see being successful are perceived as similar in terms of ability or background. This makes sense in the context of DBG, as most of the students come from similar backgrounds and face 
similar challenges. Seeing someone like them being successful, therefore, allowed the participants to believe they had the potential for success as well.

These findings suggest that programs may want to prioritize peer modeling and ensure that students are made aware of each other's successes. This could be achieved by instituting a purposeful peer-mentoring program, creating a "wall of success" that students and staff can add to, or dedicating time in community meetings for students to share their achievements so other students are exposed to a wide range of vicarious experiences. Establishing the previously mentioned student advisory board would be another way to highlight student success while also encouraging students to take up leadership roles.

\section{Study Limitations and Conclusion}

The methods, participants, and researchers introduced possible limitations to the study. These data were collected as part of an ongoing evaluation of the gym that is, in part, aimed at examining whether and how program effects are changing over time. As such, focus groups were conducted at two time points, which were separated by nearly three years. Combining such data could certainly introduce a number of limitations, though in the current study the themes were found to cut across all of the focus groups, regardless of when the data were collected. Regarding the participants, although the authors attempted to gather a representative sample of gym participants, our ultimate sample was largely determined by attendance, which may have affected study findings. In addition, it is also possible that staff members selected students to participate whom they were either more familiar with or whom they believed might paint a more positive picture of the gym, which could have shaped study findings.

In addition to limitations introduced by the methods and study sample, researcher presumptions may also have affected the results. Prior to conducting the focus group interviews, the research team was already aware of the success of the program, which may have primed us to structure our interview questions and hear student responses in a way that magnified the impact the gym was having on students' self-efficacy beliefs. Similarly, we as researchers were also primed to interpret student responses as fitting into Bandura's four previously defined ways of developing self-efficacy. Although we intentionally looked for instances where student narratives deviated from Bandura's theory, it is possible that our interpretations were constrained due to the theoretical grounding of the study. 
Despite these limitations, results of the current study point to specific ways DBG is positively impacting the self-efficacy beliefs of the lower-income youth of color in the program. Research that identifies successful mechanisms of self-efficacy development in the context of communitybased programs has been largely absent from the research literature. Findings from the current study, therefore, outline some specific steps youth development programs can take to support the self-efficacy development of students who may otherwise be excluded from efficacybuilding experiences as a result of systemic inequalities.

\section{References}

Alivernini, F., \& Lucidi, F. (2011). Relationship between social context, self-efficacy, motivation, academic achievement, and intention to drop out of high school: A longitudinal study. The Journal of Educational Research, 104(4), 241-252. doi:10.1080/00220671003728062

Bandura, A. (1977). Self-efficacy: Toward a unifying theory of behavioral change. Psychological Review, 84(2), 191-215. doi:10.1037/0033-295X.84.2.191

Bandura, A. (1982). Self-efficacy mechanism in human agency. American Psychologist, 372), 122-147. doi:10.1037/0003-066X.37.2.122

Bandura, A. (1997). Self-Efficacy: The Exercise of Control. New York, NY: Freeman.

Bandura, A., Barbaranelli, C., Caprara, G.V., \& Pastorelli, C. (2001). Self-efficacy beliefs as shapers of children's aspirations and career trajectories. Child Development, 72(1), 187-206.

Bandura, A., \& Cervone, D. (1983). Self-evaluative and self-efficacy mechanisms governing the motivational effects of goals systems. Journal of Personality and Social Psychology, 45(5), 10171028. doi: $10.1037 / 0022-3514.45 .5 .1017$

Berg, M., Coman, E., \& Schensul, J. (2009). Youth action research for prevention: A multi-level intervention designed to increase efficacy and empowerment among urban youth. American Journal of Community Psychology, 43(3-4), 345-359. doi:10.1007/s10464-009-9231-2

Braun, V., \& Clarke, V. (2006). Using thematic analysis in psychology. Qualitative Research in Psychology, 3(2), 77-101. doi:10.1191/1478088706qp063oa

Britner, S. L., \& Pajares, F. (2006). Sources of science self-efficacy beliefs of middle school students. Journal of Research in Science Teaching, 43(5), 485-499. doi:10.1002/tea.20131

Butz, A. R., \& Usher, E. L. (2015). Salient sources of early adolescents' self-efficacy in two domains. Contemporary Educational Psychology, 42, 49-61. doi:10.1016/j.cedpsych.2015.04.001

Cervone, D. (1997). Social-cognitive mechanisms and personality coherence: Self-knowledge, situational beliefs, and cross-situational coherence in perceived self-efficacy. Psychological Science, $8(1)$, 43-50. doi:10.1111/j.1467-9280.1997.tb00542.x 
Journal of Youth Development | http://jyd.pitt.edu/ | Vol. 14 Issue 1 DOI 10.5195/jyd.2019.699

Improving Self-Efficacy Via Community Programs

Darling-Hammond, L. (2001). Unequal Opportunity: Race and Education. Retrieved from https://www.brookings.edu/articles/unequal-opportunity-race-and-education/

DeAngelis, T. (2012). Helping at-risk students succeed: A psychologist-designed program that supports learning among at-risk kids gains momentum nationwide. Monitor on Psychology, 43(2), 46-47.

Dee, T., \& Gershenson, S. (2017). Unconscious Bias in the Classroom: Evidence and Opportunities. Mountain View, CA: Google Inc. Retrieved from https://goo.gl/O6Btqi

Devine, P. G., Forscher, P. S., Austin, A. J., \& Cox, W. T. L. (2012). Long-term reduction in implicit race bias: A prejudice habit-breaking intervention. Journal of Experimental Social Psychology, 48(6), 1267-1278. doi:10.1016/j.jesp.2012.06.003

Grether, T., Sowislo, J. F., \& Wiese, B. S. (2018). Top-down or bottom-up? Prospective relations between general and domain-specific self-efficacy beliefs during a work-family transition. Personality and Individual Differences, 121, 131-139. doi:10.1016/j.paid.2017.09.021

Jussim, L., \& Harber, K. D. (2005). Teacher expectations and self-fulfilling prophecies: Knowns and unknowns, resolved and unresolved controversies. Personality and Social Psychology Review, g(2), 131-155

Morton, M. H., \& Montgomery, P. (2013). Youth empowerment programs for improving adolescents' selfefficacy and self-esteem: A systematic review. Research on Social Work Practice, 23(1), 22-33. doi:10.1177/1049731512459967

Nickens, H. W., \& Smedley, B. D. (2001). The right thing to do, the smart thing to do: Enhancing diversity in the health professions: Summary of the Symposium on Diversity in Health Professions in Honor of Herbert W. Nickens, M.D. Washington, DC: National Academy Press. Retrieved from https://www.nap.edu/catalog/10186/the-right-thing-to-do-the-smart-thing-to-do

Robbins, S. B., Lauver, K., Le, H., Davis, D., Langley, R., \& Carlstrom, A. (2004). Do psychosocial and study skill factors predict college outcomes? A meta-analysis. Psychological Bulletin, 130, 261288. doi:10.1037/0033-2909.130.2.261

Samuels, S. M., \& Gibb, R. W. (2002). Self-efficacy assessment and generalization in physical education courses. Journal of Applied Social Psychology, 32(6), 1314-1327. doi:10.1111/j.15591816.2002.tb01438.x

Schunk, D. H., \& Pajares, F. (2002). The development of academic self-efficacy. In A. Wigfield \& J. S. Eccles (Eds.), Development of Achievement Motivation (pp. 15-31). San Diego: Academic Press. doi:10.1016/B978-012750053-9/50003-6

Schwartz, S., \& Suyemoto, K. (2013). Creating change from the inside: Youth development within a youth community organizing program. Journal of Community Psychology, 41(3), 341-358. doi:10.1002/jcop.21541 
Journal of Youth Development | http://jyd.pitt.edu/ | Vol. 14 Issue 1 DOI 10.5195/jyd.2019.699

Improving Self-Efficacy Via Community Programs

Schwarzer, R., \& Jerusalem, M. (1995). Generalized self-efficacy scale. In J. Weinman, S. Wright, \& M. Johnston (Eds.), Measures in Health Psychology: A User's Portfolio (pp. 35-37). Windsor, UK: NFER-NELSON.

Shelton, S. H. (1990). Developing the construct of general self-efficacy. Psychological Reports, 66, 987994. doi:10.1177/003329419006600301

Usher, E. L., \& Pajares, F. (2008). Sources of self-efficacy in school: critical review of the literature and future directions. Review of Educational Research, 78(4), 751-796. doi:10.3102/0034654308321456

Vaismoradi, M., Turunen, H., \& Bondas, T. (2013). Content analysis and thematic analysis: Implications for conducting a qualitative descriptive study. Nursing and Health Sciences, 15, 398-405. doi:10.1111/nhs. 12048 